\title{
Follicular Lymphoma Involving Bilateral Ovaries Following Routine Hysterectomy and Bilateral Salpingo-Oophorectomy: An Incidental Finding
}

Anoshia Afzal*, Michael Quinton*, Umar Farooque^, Bilal Ahmed Khan^, Sepideh Asadbeigi**, Shahbaz Khan***

*Department of Pathology, University of Oklahoma Health Sciences Center, Oklahoma City, OK 73104, USA.

* Correspondence: anoshia.afzal@ hotmail.com

${ }^{\wedge}$ Department of Internal Medicine, Dow Medical College, Dow University of Health Sciences • Karachi, Pakistan

**Department of Dermatology, McGaw Medical Center of Northwestern University, Chicago, IL, USA.

***Gastrointestinal, Hepatobiliary and Transplant Pathology, Indiana University School of Medicine · Indianapolis, USA.

Conflicts of interest: None

Funding: None

Acknowledgements: None

Author contributions: All authors have made substantial contributions to the preparation of manuscript with the most writing being done by the first author.

*No competing interests to declare.

*No identifying information is present in the case report. The patient was informed and verbal consent was taken that a de-identified case report will be published. There is no personal information present in the case report.

\section{$\underline{\text { Abstract }}$}

Ovarian lymphoma is an infrequent disease, accounting for less than $1 \%$ of all non-Hodgkin lymphoma diagnosis. Symptoms include abnormal vaginal bleeding or discharge, abdominal pain, and urinary obstruction due to the large mass. In our case, a 60-year-old woman, underwent a total abdominal hysterectomy and bilateral salpingo-oophorectomy, as she presented with lowgrade follicular lymphoma (FL) in both the ovaries and the left ovary was observed to be enlarged. The tumor is categorized as lymphoma based upon immunohistochemical markers. Computed tomography (CT) scan of the chest, abdomen, and pelvis and bone marrow biopsy are important for the staging of primary lymphoma of the ovary. The first-line chemotherapy 
regimen includes rituximab, cyclophosphamide, doxorubicin hydrochloride (hydroxydaunorubicin), vincristine sulfate (Oncovin), and prednisone (R-CHOP) for rapidly proliferative non-Hodgkin lymphoma (NHL). Lymphomas with slower growth patterns can be treated with Bendamustine-Rituximab and don't need aggressive R-CHOP treatment.

Keywords: lymphoma, female genital tract, r-chop, non-hodgkin's lymphoma, follicular lymphoma, ovarian lymphoma, ovary, lymphoma, chemotherapy.

\section{Introduction}

Non-Hodgkin lymphoma (NHL) includes B-cell and T-cell lymphomas. NHL is the seventh most common malignancy [1]. When considering its ovarian involvement, the NHL has a prevalence of $1.5 \%$ amid all ovarian neoplasm and 0.5-1\% amid all NHL. Ovarian lymphoma, an uncommon disease, is even rarer as a primary lymphoma, whereas secondary involvement of the ovary can follow in the late stage of NHL [2]. It is very tough to differentiate this condition from uterine fibroids or sarcoma. Likewise, non-specific symptoms lead to delay in diagnosis. Symptoms include abnormal vaginal bleeding or discharge, abdominal pain, and urinary obstruction due to the large mass. Usually, NHL involving the uterus is a low-stage neoplasm. Primary presentation of Lymphoma presenting with ovarian mass is extremely rare, owing to just $0.3 \%$ incidence [3]. We present a case of low-grade follicular lymphoma (FL) involving bilateral ovaries following routine hysterectomy and bilateral salpingo-oophorectomy in a 60-year-old woman who presented with vague left-sided pelvic pain.

\section{Case presentation}

Our patient was a 60-year-old female, known case of type II diabetes mellitus, hypertension, and morbid obesity, who was referred to us from an outside hospital with complains of vague leftsided pelvic pain and abdominal discomfort for three months. Ultrasound revealed an enlarged and cystic left ovary, which was found to be 6.0 x $4.0 \times 3.5 \mathrm{~cm}$ grossly. Keeping all the features under consideration including age and risk factors, abdominal hysterectomy and bilateral salpingo-oophorectomy were performed on the patient. Serosa of the fallopian tubes contained small nodular deposits. Histologic sections of both ovaries revealed a low-grade lymphoid population with nodular architecture (Figures 1A-1C). The lymphocytic cell population was found to be positive for a CD10 (Figure 1D) with co-expression of B-cell lymphoma-2 (Bcl-2) and Bcl-6. CD20 (Figure 2A) was positive along with focally preserved CD23 (Figure 2B) in some nodular areas. Some areas showed a high proliferation index of about 30-40\% by Ki-67 (Figure 2C); however, no large cell morphology was seen. Bcl-2/immunoglobulin IgH rearrangement came positive on fluorescence in situ hybridization (FISH). The final diagnosis rendered was follicular lymphoma involving bilateral ovaries. Bone marrow biopsy was done and it revealed active trilineage hematopoieisis with no evidence of involvement by follicular lymphoma. The patient returned to her primary facility for further treatment and management. 


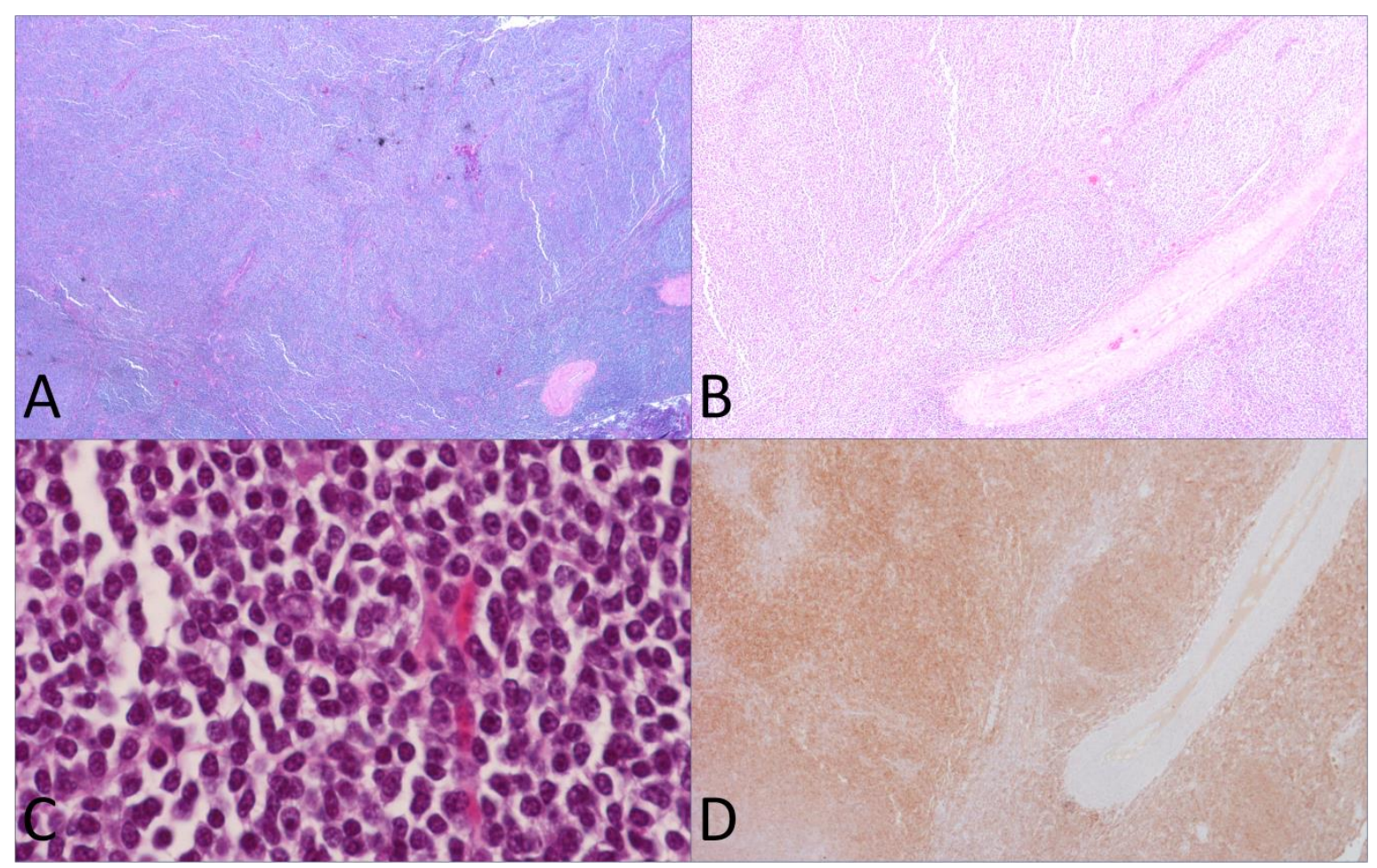

Figure 1: Hematoxylin and eosin sections reveal low-grade disease with predominantly nodular architecture (A) with some areas showing diffuse infiltration [B (10x) and C (20x)]. These Bcells were expressing CD10 (D).

CD- cluster of differentiation 


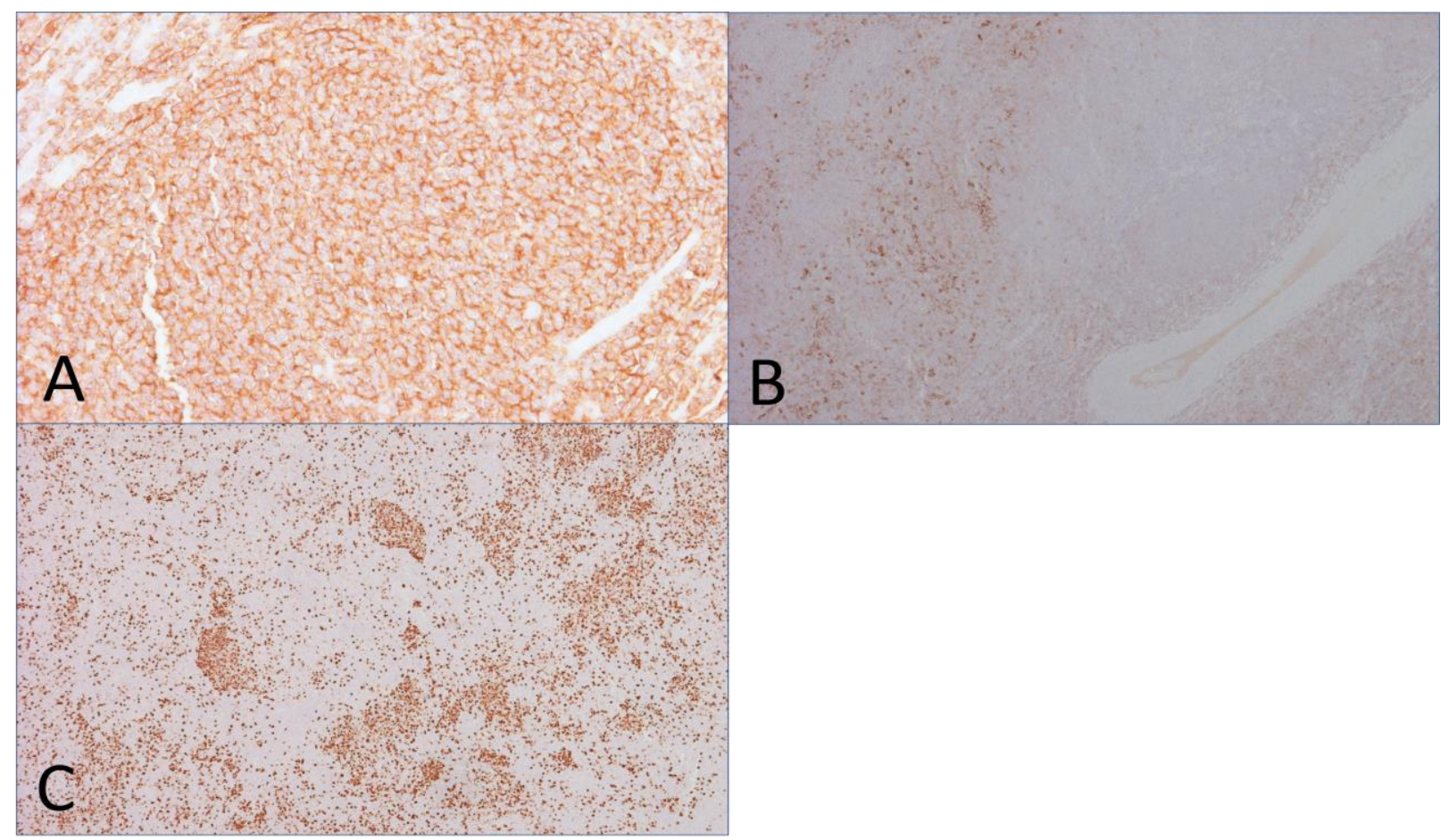

Figure 2: Immunohistochemistry shows CD20 positive B-cells (A), which were also expressing CD23 (B) in some areas. Focal increase of the proliferation index by Ki-67 (C) by about 30-40\% was seen in certain areas.

\section{Discussion:}

Ovarian lymphomas can arise at any age but most frequently in the fourth decade of life. Abdominal pain and distention, ascites, abdominal or pelvic mass, men- constitutional symptoms like bladder and bowel discomfort or irregular vaginal bleeding are the most frequent symptoms [4]. 41-71\% of ovarian lymphomas are reported to be present bilaterally. Ovarian lymphomas having clinical findings very alike ovarian malignancies of epithelial origin are the toughest to assume pre-operatively [5]. According to diagnostic criteria of primary ovarian lymphoma proposed by Fox et al., lymphoma should be limited to the ovary, with direct spread to the adjacent lymph nodes or structures, bone marrow aspiration, and peripheral blood smear should be negative, and metastatic involvement should be seen at least after months [6]. The difference between ovarian and extra-ovarian lymphoma is that the tumor cells stick to reticulin to form pseudo-acini and can grow in cords or nests in ovarian lymphoma [7]. B-cell NHL is the more frequently occurring lymphoma of both B-cell \& T-cell NHL that occurs in the ovary. It stainpositive for B-cell marker CD20 and stays negative for T-lineage marker CD3 [8]. Immunohistochemical markers classify the tumor as lymphomas. This can be determined by chloracetate esterase or myeloperoxidase staining [7]. Histological specimen with positive staining for leukocyte common antigen (LCA) differentiates malignant lymphoma from nonlymphoid neoplasm. Computed tomography (CT) scan of the chest, abdomen, and pelvis and 
bone marrow biopsy are important for the staging of primary lymphoma of the ovary [9]. Staging $\&$ therapeutic assessment is also done through Positron Emission Tomography (PET) with 18FFlouro-Deoxy-Glucose (FDG) [8]. Furthermore, histopathological stains like hematoxylin and eosin stain (H\&E) will also show the characteristic features of B-cell lymphoma. Translocation between c-MYC oncogene on chromosome eight and frequently the heavy chain gene on chromosome 14 occurs in all forms of B-cell lymphoma [10]. Ferrozzi et al. studied the imaging patterns shared by all his eight reported cases with ovarian NHL and concluded that the lymphomas were always greater than $5 \mathrm{~cm}$ in diameter, bilateral, homogenous, and not associated with ascites [11]. Ultrasonography revealed homogenous, hypoechoic, and slightly vascularized tumors. CT scan showed retinal bleeding and dilated subarachnoid space around the optic nerve bilaterally. Primary ovarian lymphoma (POL) presents early and is diagnosed at an early stage so has a better survival rate of $80 \%$ as compared to $33 \%$ of secondary cases. Treatment is mainly based on classifying the patients into high-risk and low-risk groups according to the subsequent conditions: tumor size of $10 \mathrm{~cm}$ or more, disease stage [bone marrow extension and central nervous system (CNS) penetration], performance status of the patient, and elevation of lactate dehydrogenase [9]. Pelvic NHL is very responsive to chemotherapy and doesn't require surgical staging and cytoreduction. Pre-operative biopsies can help in making a diagnosis [5-9].

\section{Conclusions}

Using criteria proposed in past studies, it cannot be definitively determined if this presentation represents primary ovarian follicular lymphoma, however, the discovery of this low-grade lymphoma following a routine gynecologic surgery highlights the importance of considering the diagnosis in the setting of ovarian tumors.

\section{$\underline{\text { References }}$}

1. National Cancer Institute| Surveillance, Epidemiology, and End Results Program. (2016). Accessed: July 10, 2020: http://seer.cancer.gov/.

2. Elharroudi T, Ismaili N, Errihani H, Jalil A: Primary lymphoma of the ovary. J Cancer Res Ther. 2008, 4:195-6. 10.4103/0973-1482.44291

3. Malempati LM, Nandan N, Babu S: Case of extranodal non-hodgkins lymphoma involving the endometrium and the ovaries: a rare case report. Int J Reprod Contracept Obstet Gynecol. 2017, 6:4131-34. 10.18203/2320-1770.ijrcog20174076

4. Kumar N, Yadav A: Bilateral primary ovarian large B cell non-hodgkin's lymphoma: a rare case report. Indian J Gynecol Oncolog. 2019, 17:42. 10.1007/s40944-019-0283-4

5. Kumar N, Kumar R, Bera A, Srinivasan R, Sharma SC: Primary ovarian lymphoma: a case report and review of literature. J Obstet Gynaecol India. 2014, 64:65-7. 10.1007/s13224-012-0200-6

6. Fox H, Langley FA, Govan AD, Hill AS, Bennett MH: Malignant lymphoma presenting as an ovarian tumour: a clinicopathological analysis of 34 cases. Br J Obstet Gynaecol . 1988, 95:386-90. 10.1111/j.1471-0528.1988.tb06611.x 
7. Bhartiya R, Kumari N, Mallik M, Singh RVN: Primary non-hodgkin's lymphoma of the ovary - a case report. J Clin Diagn Res. 2016, 10:ED10-11. 10.7860/JCDR/2016/19346.7766

8. Komoto D, Nishiyama Y, Yamamoto Y, et al.: A case of non-hodgkin's lymphoma of the ovary: usefulness of 18F-FDG PET for staging and assessment of the therapeutic response. Ann Nucl Med. 2006, 20:157-60. 10.1007/BF02985629

9. Vang R, Medeiros LJ, Fuller GN, Sarris AH, Deavers M: Non-hodgkin's lymphoma involving the gynecologic tract: a review of 88 cases. Adv Anat Pathol. 2001, 8:200-17. 10.1097/00125480-200107000-00002

10. Al-Maghrabi H, Meliti A: Primary bilateral ovarian burkitt lymphoma; a rare issue in gynecologic oncology. J Surg Case Rep. 2018, 2018:rjy113. 10.1093/jscr/rjy113

11. Ferrozzi F, Catanese C, Uccelli M, Bassi P: Ovarian lymphoma. Findings with ultrasonography, computerized tomography and magnetic resonance. Radiol Med. 1998, 95:493-7. 